\title{
Commentary: Covid-19 Pneumonitis and Cystic Lung Disease, Pneumothorax and Pneumomediastinum
}

\author{
Serenydd Everden, Jack West
}

Department of Emergency Medicine, Coffs Harbour Base Hospital, Coffs Harbour, NSW, Australia

Article Info

\section{Article Notes}

Received: November 11, 2021

Accepted: December 27, 2021

\section{${ }^{*}$ Correspondence:}

Dr. Serenydd Everden, Department of Emergency Medicine, Coffs Harbour Base Hospital, Coffs Harbour, NSW, Australia; Email: serenydd.everden@googlemail.com

Dr. Jack West, Department of Emergency Medicine, Coffs Harbour Base Hospital, Coffs Harbour, NSW, Australia; Email: fordjwest@gmail.com

(c) 2021 Everden S, West J. This article is distributed under the terms of the Creative Commons Attribution 4.0 International License.

\section{Keywords}

Covid-19

SARS-CoV-2

Pneumonitis

Pneumothorax

Mediastinal Emphysema

Cysts
Cysts in covid-19 are considered an atypical CT finding, with cystic changes occurring in up to $10 \%$ of covid-19 patients ${ }^{1}$. Yet an increasing body of evidence points to the clinical relevance of cystic changes secondary to covid $-19^{2}$. Subsequently there is a need to increase awareness and management of their potential sequelae.

Cyst related complications in covid-19 tend to present later in the disease course, and this matches the findings of late stage ARDS caused by SARS ${ }^{3}$. CT findings in late stage ARDS suggests that severe SARS-induced ARDS may independently result in cyst formation ${ }^{3}$. Joynt et al. summarized the radiological findings into two groups as follows:

- Early stage of ARDS: ground glass opacities and consolidation

- Late phase of ARDS: fibrotic interlobulated septae, parenchymal bands, honeycombing and cyst development

Covid-19 has been seen to mirror these some of these ARDS findings. Martinelli et al repeatedly observed cyst formation as a late finding in covid-19 pneumonia patients ${ }^{2}$. Patients often show signs of improvement one to three weeks into the disease course ${ }^{4}$. In the clinical experience of the authors, many individuals are approaching or past the point of discharge by this stage. We argue therefore, that if a patient is known to have extensive cystic changes on CT, it is important to consider that they may deteriorate rapidly post discharge secondary to a complication from cyst rupture. Complications may include, pneumatoceles ${ }^{5}$, pneumothoraces, and pneumomediastinums ${ }^{2,6}$.

Cystic CT changes in the context of covid-19 pneumonia have been observed to progress into pneumatoceles, pneumothoraces, and more rarely pneumomediastiums ${ }^{2,6}$, with approximately $1 \%$ of covid-19 infections developing pneumothoraces ${ }^{2}$. However, it is important to acknowledge that the majority of cysts remain asymptomatic and are not (yet) known to result in long term complications. The small percentage of cysts that lead to complications remain important to understand, as the complications may be life-threatening. The pathogenesis of cyst formation may be related to ischaemic parenchymal damage and inflammation ${ }^{3}$. Rupture, either spontaneous or secondary to barotrauma from ventilation or expectoration, followed by tissue dissection via the Macklin effect is hypothesised to constitute the mechanism for this progression. Traditionally these complications have all been managed conservatively, with the exception of large pneumothoraces requiring chest drain insertion. All of the above complications 
have implications for the application of positive pressure ventilation whether by Continuous Positive Airway Pressure (CPAP) or Endotracheal Intubation. The significant challenges posed by the covid-19 Pandemic and the novel nature of the disease have resulted in trials of more invasive management for cysts and cyst related complications. For example, pig-tail catheter drainage of pneumatoceles ${ }^{7}$. However, as yet there remains insufficient evidence base to suggest whether their widespread use would be superior to conservative management alone ${ }^{8}$. We need a unified evidence-based approach, and need to encourage discussion surrounding management of cysts in covid-19.

It is interesting to postulate whether cystic formation is related to greater disease severity more broadly. One significant difficulty in drawing conclusions in this regard is that mild covid-19 cases generally avoid hospitalization, let alone the CT imaging that would be necessary to quantify cyst formation. In order to draw concrete inferences, it would be necessary to image relatively well patients, or to identify a sub section incidentally receiving CT imaging for alternative indications.

In summary, significant circumstantial evidence for covid-19 cystic changes associating with complications such as pneumatocele, pneumothorax, and pneumomediastinum exists. The mechanism for such changes is theoretically sound, but unconfirmed. The primary aim of this commentary is to raise awareness of these complications, thus expediting their diagnosis and appropriate management in covid-19 patients. This is especially pertinent given how as later stage complications, they may coincide with patients who are otherwise clinically improving, or already discharged from hospital. Further research of Covid-19 disease course and long term follow up is required, ideally in the form of multicenter collaborative studies, which include multivariate analysis of risk factors such as age, smoking history, and treatment interventions. Currently, there is scant specific evidence to suggest direct invasive management of cysts or pneumatoceles prior to their potential development into pneumothorax or pneumomediastinum. However, there are pre-existing bodies of evidence to guide the general management of both conditions, and as authors these are what we advise $\mathrm{e}^{9,10}$.

\section{Acknowledgement}

Produced without financial support, grants or funding.

\section{Conflict of interest}

No competing interests to disclose.

\section{References}

1. Shi H, Han X, Jiang N, et al. Radiological findings from 81 patients with COVID-19 pneumonia in Wuhan, China: a descriptive study. Lancet Infect Dis. 2020; 20(4): 425-434. doi:10.1016/S14733099(20)30086-4/ATTACHMENT/D9FABEDA-1DE4-4419-A7A03E2DFCDACBC4/MMC2.PDF

2. Martinelli AW, Ingle T, Newman J, et al. COVID-19 and pneumothorax: A multicentre retrospective case series. Eur Respir J. 2020; 56(5). doi:10.1183/13993003.02697-2020

3. Joynt GM, Antonio GE, Lam P, et al. Late-Stage Adult Respiratory Distress Syndrome Caused by Severe Acute Respiratory Syndrome: Abnormal Findings at Thin-Section CT1. 2004; 230(2): 339-346. doi:10.1148/RADIOL.2303030894

4. Aguilar RB, Hardigan P, Mayi B, et al. Current Understanding of COVID-19 Clinical Course and Investigational Treatments. Front Med. 2020; 7: 638. doi:10.3389/FMED.2020.555301/BIBTEX

5. Sugimoto H, Era Y, Sugimoto K. Pneumatocele after recovering from COVID-19. BMJ Case Reports CP. 2021; 14(4): e242729. doi:10.1136/ BCR-2021-242729

6. Everden S, Zaki I, Trevelyan G, et al. COVID-19 pneumonitis and cystic lung disease, pneumothorax and pneumomediastinum. Thorax. Published online 2021. doi:10.1136/THORAXJNL-2021-217390

7. Brahmbhatt N, Tamimi O, Ellison H, et al. Pneumatocele and cysts in a patient with severe acute respiratory syndrome coronavirus 2 infection. JTCVS Tech. 2020; 4: 353. doi:10.1016/J.XJTC.2020.08.047

8. Ramzy D. Commentary: Pneumatocele and cysts in a patient with severe acute respiratory syndrome coronavirus-2 (SARSCoV-2) infection-yet another new complication associated with coronavirus disease 2019 (COVID-19). JTCVS Tech. 2020; 4: 358-359. doi:10.1016/J.XJTC.2020.09.012

9. MacDuff A, Arnold A, Harvey J. Management of spontaneous pneumothorax: British Thoracic Society pleural disease guideline 2010. Thorax. 2010; 65(Suppl 2): ii18-ii31. doi:10.1136/ THX.2010.136986

10. C Hyzy R, Taha AR. Diagnosis, management, and prevention of pulmonary barotrauma during invasive mechanical ventilation in adults. Up to Date. Published 2016. Accessed November 12, 2021. https://www.uptodate.com/contents/diagnosis-managementand-prevention-of-pulmonary-barotrauma-during-invasivemechanical-ventilation-in-adults?search=pneumomediastinum management\&source=search_result\&selectedTitle=2 109\&usage type $=$ default $\&$ display_rank $=2$ 